\title{
Defective Polymorphonuclear Leukocyte Formyl Peptide Receptor(s) in Juvenile Periodontitis
}

\author{
H. Daniel Perez, ${ }^{\star}$ Edward Kelly, ${ }^{*}$ Fred Elfman, ${ }^{*}$ Gary Armitage, ${ }^{\star}$ and James Winkler ${ }^{*}$ \\ ${ }^{*}$ Rosalind Russell Arthritis Research Laboratory, Department of Medicine, and ${ }^{\ddagger}$ Department of Stomatology, \\ School of Dentistry, University of California, San Francisco, California 94143; and The Medical Service, \\ San Francisco General Hospital, San Francisco, California 94110
}

\begin{abstract}
Juvenile periodontitis (JP) is a disease characterized by severe gingival infections. PMN from some JP patients exhibit abnormal chemotactic responsiveness when challenged with the synthetic formyl peptide, FMLP. While investigating PMN function in JP, we found a patient in whom abnormal PMN chemotactic responses to FMLP were associated with a defective population of PMN formyl peptide receptor(s) (FPR). JP PMN failed to respond chemotactically when challenged with FMLP, but exhibited normal chemotactic responses upon exposure to purified human C5a. Furthermore, JP PMN were capable of degranulating and generating superoxide anion radicals as well as normal PMN upon exposure to FMLP. Binding studies demonstrated that JP PMN had a diminution in the number of high-affinity FPR. Studies in which FPR was radiolabeled by chemical cross-linking demonstrated that JP PMN FPR exhibited the same molecular weight and $\boldsymbol{N}$-linked glycosylation as normal PMN FPR. JP PMN FPR, however, was more resistant to papain cleavage than normal PMN FPR. Autoradiograms obtained from 2D-PAGE of normal and JP PMN FPR demonstrated decreased amounts of FPR isoforms in JP PMN. (J. Clin. Invest. 1991. 87:971-976.) Key words: neutrophil $\bullet$ chemotaxis $\bullet$ degranulation $\bullet$ binding $\bullet$ cross-linking
\end{abstract}

\section{Introduction}

Exposure of PMN to $N$-formyl peptides stimulates these cells to migrate in a directed fashion (i.e., respond chemotactically) (1), selectively release a portion of their lysosomal contents (i.e., degranulation) (1), and generate highly reactive oxygen-derived free radicals (such as superoxide anion) (2). These processes are initiated by the binding of formyl peptides to specific receptors present on the PMN membrane (3).

Juvenile periodontitis (JP) ${ }^{1}$ is a disease characterized by severe (and protracted) gingival infections, leading to tooth loss

Address correspondence to Dr. H. Daniel Perez, Building 30, Room 3300, San Francisco General Hospital, 1001 Potrero Avenue, San Francisco, CA 94110.

Received for publication 2 January 1990 and in revised form 2 October 1990

1. Abbreviations used in this paper: EGS, ethylene glycolbis succinimidyl succinate; FP, $N$-formyl-Nleu-leu-phe-tyr; FPR, formyl peptide receptors; JP, juvenile periodontitis.

J. Clin. Invest.

(c) The American Society for Clinical Investigation, Inc.

0021-9738/91/03/0971/06 \$2.00

Volume 87, March 1991, 971-976
(4). PMN from some patients with JP exhibit abnormal chemotactic responsiveness when challenged with the synthetic chemotactic peptide, FMLP (5). Van Dyke et al. (6) reported that PMN from some JP patients exhibit a diminution in their ability to bind ${ }^{3} \mathrm{H}$-FMLP (i.e., decreased receptor number). In the course of studying JP PMN responses to chemotactic factors, we have found a patient in whom abnormal PMN chemotactic responsiveness to formyl peptide is associated with a defective population of formyl peptide receptors (FPR).

\section{Methods}

Patient. The JP patient was a 22-yr old Caucasian male selected from patients seen at the Stomatology Clinical Research Center at the University of California, San Francisco, and gave informed consent to participate in this study. The initial examination revealed radiographic evidence of bilateral symmetrical severe angular bony defects on the maxillary and mandibular first permanent molars and mandibular central incisors. Attachment loss in all molar regions was $>8 \mathrm{~mm}$ and $>6 \mathrm{~mm}$ in the incisor region. Bleeding on probing was present at these sites. All other teeth had attachment loss of $<3 \mathrm{~mm}$ and no bleeding on probing. Medical examination and standard blood work-up were within normal limits. DNA probe analysis of the afflicted sites demonstrated high levels of Haemophilus (Actinobacillus) actinomycetemcomitans, Bacteroides intermedius, and Eikenella corrodens. A diagnosis of juvenile periodontitis was made based on the clinical, radiographic, and microbiological features of the case (7). It should be noted that, at the time of study, the patient was off antibiotic therapy and in the chronic phase of JP. At this stage, PMN do not predominate in the oral lesions ( 7 ).

Assays of PMN function. Platelet-poor leukocyte suspensions containing $97-99 \%$ PMN were prepared from venous blood $(50 \mathrm{ml})$ that had been mixed with $12 \mathrm{ml}$ of acid-citrate dextrose, as described (8). Stimulated random motility (chemokinesis) and directed migration (chemotaxis) of PMN were measured using a minor modification (9) of the leading front method of Zigmond and Hirsch (10). Results are expressed as the distance $(\mu \mathrm{m} / 35 \mathrm{~min})$ that the leading front of cells migrated into $3.0-\mu \mathrm{m}$ pore diameter cellulose nitrate micropore filters (Sartorious Filters, Inc., Hayward, CA) separating the upper, or cell compartments, from the lower, or stimulus compartments, of modified Boyden chambers. Chemotaxis (net migration) was calculated by subtracting stimulated random motility from total migration. Duplicate chambers were used in each experiment and five fields examined in each filter. Extracellular release of the granule markers lysozyme and beta-glucuronidase from cytochalasin B-treated PMN was assessed as described previously (8). Generation of superoxide anion ( $\mathrm{nmol} / 5 \mathrm{~min}$ per $10^{6} \mathrm{PMN}$ ) was determined using ferricytochrome $\mathrm{c}$, as described (8).

Binding of formyl peptide to $P M N$. $N$-formyl-Nleu-leu-phe-tyr (FP) (Sigma Chemical Co., St. Louis, MO) was radioiodinated with carrierfree $\mathrm{Na}^{125} \mathrm{I}$ (Amersham Corp., Arlington Heights, IL) by the chloramine $\mathrm{T}$ method, as described previously $(11,12)$. Sp act of ${ }^{125} \mathrm{I}-\mathrm{FP}$ was $\sim 600 \mathrm{Ci} / \mathrm{mmol}$. Binding of ${ }^{125} \mathrm{I}-\mathrm{FP}$ to $\mathrm{PMN}$ was assessed as described previously $(8,12)$. Briefly, PMN $\left(2.0 \times 10^{6}\right.$ cells $)$ and ${ }^{125} \mathrm{I}$-FP $(0.1-60$ $\mathrm{nM}$ ) were incubated at $4^{\circ} \mathrm{C}$ for $15 \mathrm{~min}$ in $0.4 \mathrm{ml}$ of phosphate 10 
mM-buffered $140 \mathrm{mM} \mathrm{NaCl}, \mathrm{pH} 7.4$ (PBS), in the presence and absence of 100 -fold excess unlabeled FP. A collection method involving centrifugation through silicone oil was used to separate free from bound peptide $(8,12)$.

Affinity-labeling of PMN FPR. PMN ( $10^{8}$ cells), suspended in PBS $(15 \mathrm{ml})$ containing $5.0 \mathrm{mM}$ EDTA, were incubated with $20 \mathrm{pmol}$ of ${ }^{125} \mathrm{I}-\mathrm{FP}$ for $15 \mathrm{~min}$ at $4^{\circ} \mathrm{C}$ to achieve equilibrium binding (8). After incubation, ethylene glycolbis succinimidyl succinate (EGS) (Pierce Chemical Co., Rockford, IL) $(0.2 \mathrm{mg} / \mathrm{ml})$ was added, and mixtures incubated for an additional $60 \mathrm{~min}$ at $4^{\circ} \mathrm{C}$ under continuous mixing conditions. At the end of incubation, PMN were pelleted by centrifugation $\left(150 \mathrm{~g}\right.$ for $\left.8 \mathrm{~min}, 4^{\circ} \mathrm{C}\right)$ and washed three times with cold buffer. Washed cells were solubilized using $0.5 \mathrm{ml}$ PBS containing $5.0 \mathrm{mM}$ EDTA, $5.0 \mathrm{mM}$ diisopropyl-fluorophosphate (DFP; Sigma), and $2.0 \%$ (wt/vol) octylglucoside (Calbiochem-Behring Corp., San Diego, CA). Detergent extracts were subjected to SDS-PAGE under reducing conditions using 7-17\% gradient slab gels (12). After SDS-PAGE, gels were dried and exposed (3-6 d) to Kodak XR-1 film (Eastman Kodak Co., Rochester, NY) $\left(-70^{\circ} \mathrm{C}\right)$, using a Cronex intensifying screen (DuPont Co., Wilmington, DE). Two-dimension PAGE (2D-PAGE) was performed as described by O'Farrell (13), with a pH range of 3.0-7.0 (14).

Enzyme treatment of PMN FPR. For deglycosylation experiments, detergent extracts of affinity-labeled normal and JP PMN FPR $(20 \mu \mathrm{g}$ total protein each) were incubated with $60 \mathrm{mU} / \mathrm{ml} \mathrm{N}$-glycosidase F (final reaction volume $30 \mu \mathrm{l})(N$-glycanase; Genzyme Corp., Boston, MA) at $37^{\circ} \mathrm{C}$ for $5 \mathrm{~h}$, under the conditions recommended by the manufacturer. After digestion, samples were run on SDS-PAGE and analyzed by autoradiography. For the papain digestion studies, detergent extracts of affinity-labeled normal and JP PMN FPR ( $40 \mu \mathrm{g}$ total protein each) were incubated at $37^{\circ} \mathrm{C}$ for $2 \mathrm{~min}$ with varying concentrations (0-20 U) of papain (Sigma Chemical Co., St. Louis, MO) in a final vol of $40 \mu \mathrm{l}$. At the end of incubation, reactions were stopped by the addition of $1.0 \mathrm{mM}$ (final concentration) cystatin (Sigma Chemical Co.). Tubes were placed on ice for $10 \mathrm{~min}$, after which samples were run on SDS-PAGE and analyzed by autoradiography.

Other reagents. FMLP was from Peninsula Laboratories, Inc., Belmont, CA. Highly purified human $\mathrm{C} 5 \mathrm{a}$ was prepared as described previously (14).

\section{Results}

Initially, we determined the ability of normal and JP PMN to respond chemotactically toward either the synthetic chemotactic peptide FMLP or the highly purified, human complementderived, chemotactic factor C5a. When incubated with FMLP $\left(10^{-9}-10^{-7} \mathrm{M}\right)$, normal PMN exhibited suboptimal $\left(10^{-9} \mathrm{M}\right)$ and optimal $\left(10^{-8} \mathrm{M}\right)$ chemotactic responses (Fig. 1 $\left.A\right)(15)$. Higher concentrations of FMLP $\left(10^{-7} \mathrm{M}\right)$ resulted in a diminished chemotactic response (Fig. $1 A$ ), a phenomenon known as deactivation $(15,16)$. Similar results were obtained when normal PMN were exposed to increasing concentrations $(0.1-$ $1.0 \mathrm{nM}$ ) of highly purified human C5a (Fig. $1 B$ ). In contrast to normal PMN, JP PMN failed to respond chemotactically upon exposure to FMLP $\left(10^{-9}-10^{-7} \mathrm{M}\right)$ (Fig. $\left.1 A\right)$. JP PMN, however, responded as well as normal PMN when challenged with $\mathrm{C5a}$ (0.1-1.0 nM) (Fig. 1 B).

Experiments were performed to determine the ability of FMLP and C5a to induce degranulation of cytochalasin Btreated normal and JP PMN (Fig. 2). JP PMN behaved as well as normal PMN in their ability to release the lysosomal enzyme markers lysozyme and beta-glucuronidase into supernatants upon challenge with either FMLP $\left(10^{-9}-10^{-6} \mathrm{M}\right)$ (Fig. $\left.2 A\right)$ or C5a (0.5-3.0 nM) (Fig. $2 \mathrm{~B}$ ). Similar results were obtained when normal and JP PMN were tested for their ability to generate superoxide anion radicals upon exposure to either FMLP

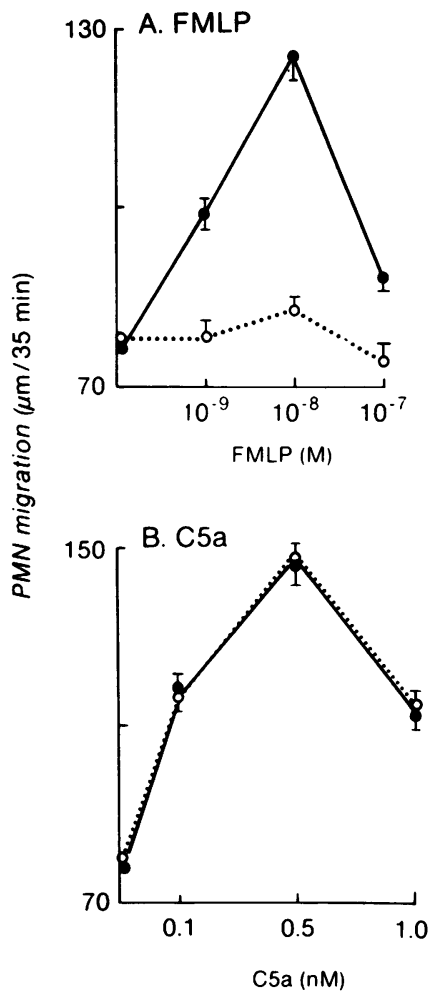

Figure 1. (A) Chemotactic responses of normal $(\bullet-\bullet)$ and JP $(\mathrm{O}-\mathrm{O})$ PMN to FMLP. (B) Chemotactic responses of normal $(\bullet-\bullet)$ and JP (O- 0 ) PMN to highly purified C5a. Results represent the mean $( \pm \mathrm{SE})$ of three experiments. $\left(10^{-9}-10^{-6} \mathrm{M}\right)$ or C5a $(0.5-3.0 \mathrm{nM})$ (in the presence and absence of cytochalasin B) (not shown). Thus, JP PMN were unable to respond chemotactically to FMLP but migrated normally toward C5a. Furthermore, JP PMN behaved as well as normal PMN with respect to their ability to degranulate and generate superoxide anion radicals upon challenge with either FMLP or C5a.

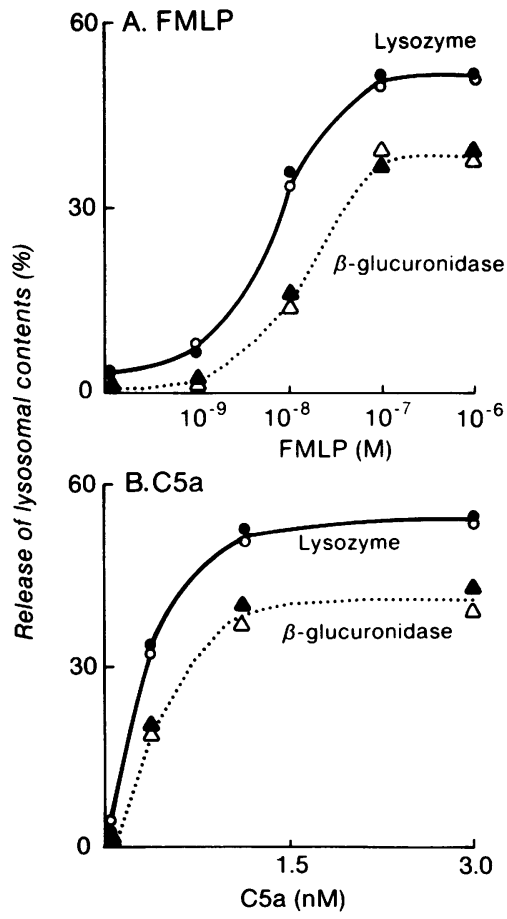

Figure 2. Release of lysosomal contents by cytochalasin B-treated normal $(\bullet-\bullet$, $\Delta-\Delta)$ and JP $(\mathrm{O}-\mathrm{O}, \Delta-\Delta)$ PMN in response to: $(A)$ increasing concentrations of FMLP; $(B)$ increasing concentrations of $\mathrm{C} 5 \mathrm{a}$. Results represent the average of two experiments performed in duplicate. 
Responses of PMN to formyl peptides are initiated by their binding to specific receptors on the cell membrane (3). Consequently, we performed experiments to determine the ability of normal and JP PMN to bind a radiolabeled analogue of FMLP, in a specific fashion. As a probe we used $N$-formyl-Nleu-leuphe-tyr- ${ }^{125}$ I ( $\left.{ }^{125} \mathrm{I}-\mathrm{FP}\right) .{ }^{125} \mathrm{I}-\mathrm{FP}$ binds to the formyl peptide receptor on human PMN (12). Binding was performed under equilibrium conditions $\left(4^{\circ} \mathrm{C}, 15 \mathrm{~min}\right)$ and results calculated as described (8). Results were best represented by curvilinear lines (Fig. 3), consistent with the presence of high and low affinity receptors (17). Normal PMN exhibited 45,586 44,818 high affinity receptors $\left(K_{d} 0.43 \pm 0.25 \mathrm{nM}\right)$ and $127,387 \pm 2,790$ low affinity receptors $\left(K_{\mathrm{d}} 2.95 \pm 1.1 \mathrm{nM}\right)$ per cell (mean $\left.\pm \mathrm{SE}, n=3\right)$. JP PMN had a diminution in the number of high affinity binding sites $\left(26,763 \pm 3,890\right.$ receptors, $\left.K_{d} 0.31 \pm 0.17 \mathrm{nM}\right)$ (mean $\pm \mathrm{SE}, n=3$ ) but exhibited a number of low affinity sites $(131,172 \pm 4,783$ receptors) (mean \pm SE, $n=3$ ) similar to that of normal cells, except that their $K_{\mathrm{d}}(5.0 \pm 0.86 \mathrm{nM})$ (mean $\pm \mathrm{SE}, n$ $=3$ ) was somewhat higher than that observed with normal PMN. Similar results (except that total receptor number was higher) were obtained when ${ }^{125} \mathrm{I}$-FP binding to normal and JP PMN was assessed using cytochalasin B-treated $\left(5 \mathrm{~min}, 37^{\circ} \mathrm{C}\right)$ cells. Furthermore, normal and JP PMN were similar in their ability to upregulate FPR (50-60\% over baseline, $5 \mathrm{~min}$ recovery at $\left.37^{\circ} \mathrm{C}\right)$ after exposure to unlabeled FP $\left(10^{-9} \mathrm{M}, 5 \mathrm{~min}\right.$, $\left.37^{\circ} \mathrm{C}\right)$.

To examine further JP PMN FPR, normal and JP PMN FPR were affinity labeled using ${ }^{125} \mathrm{I}-\mathrm{FP}$ and EGS, as described in Methods. Affinity-labeled PMN were solubilized using octylglucoside and aliquots containing identical amounts of protein were subjected to SDS-PAGE followed by autoradiography. Autoradiography of affinity-labeled normal PMN (Fig. 4, lane 1 ) revealed the presence of a single broad band exhibiting a $M_{\mathrm{r}}$ 50-66,000 D (i.e., ${ }^{125} \mathrm{I}-\mathrm{FPR}$ ) (12). Labeling was specific since it could be competed by the presence of 100 -fold excess unlabeled FP during the affinity labeling procedure (Fig. 4, lane 2). Similar results were obtained when JP PMN FPR was

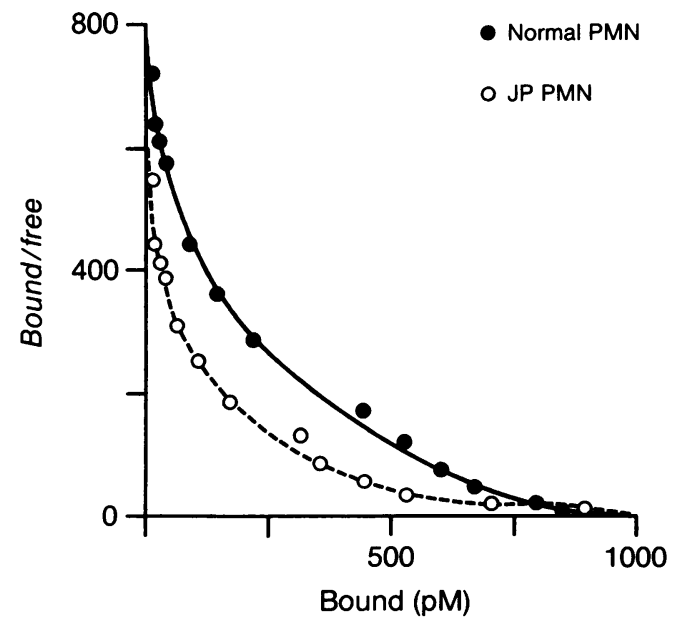

Figure 3. Scatchard plot analysis of specific binding of ${ }^{125} \mathrm{I}-\mathrm{FP}$ to normal (- - ) and JP (oincubated for $15 \mathrm{~min}$ at $4^{\circ} \mathrm{C}$ with varying concentrations of ${ }^{125} \mathrm{I}-\mathrm{FP}$, in the presence and absence of 100-fold excess unlabeled FP, and reactions terminated by centrifugation through silicone oil $(8,12)$. One of three experiments (see text).

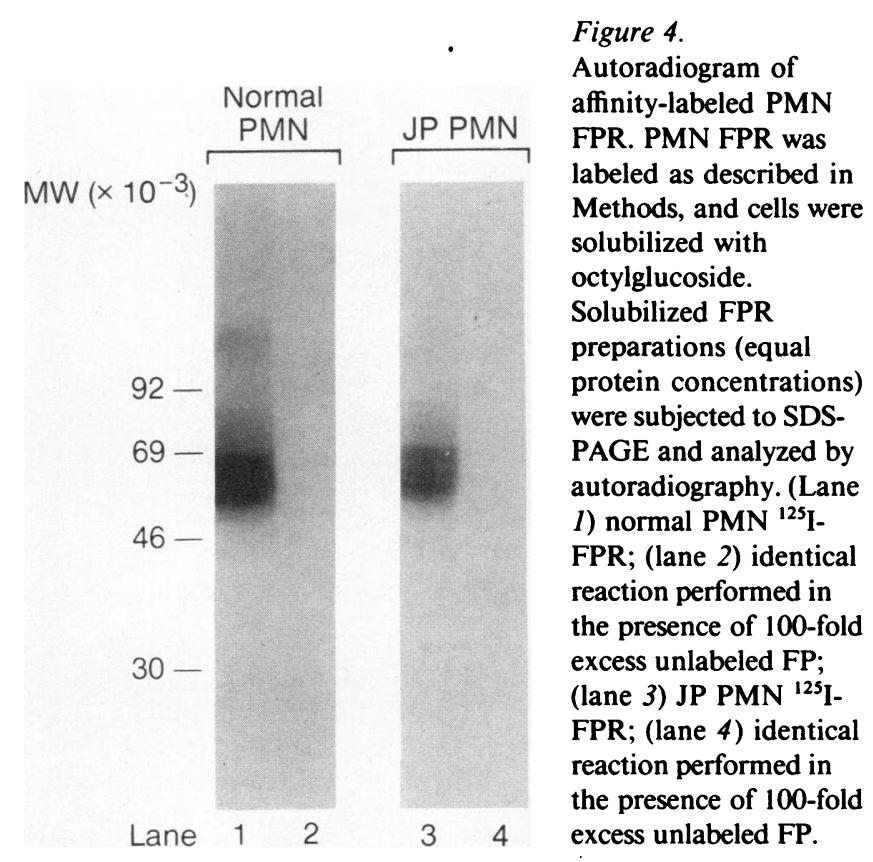

affinity labeled under identical conditions (Fig. 4, lanes 3 and 4 ).

PMN FPR is a highly glycosylated protein (18). Enzymatic removal of its asparagine-linked oligosaccharides reduces the $M_{\mathrm{r}}$ of ${ }^{125}$ I-FPR from $50-66,000 \mathrm{D}$ to $\sim 32-35,000$ (18). To determine if JP PMN FPR exhibited similar glycosylation as normal PMN FPR, we examined the ability of the enzyme $N$-glycanase to deglycosylate affinity-labeled FPR obtained from normal and JP PMN (Fig. 5). Incubation of detergent extracts obtained from affinity-labeled normal and JP PMN (20 $\mu$ g each) with $N$-glycanase at $37^{\circ} \mathrm{C}$ for $5 \mathrm{~h}$ resulted in a diminution in the $M_{\mathrm{r}}$ of ${ }^{125} \mathrm{I}-\mathrm{FPR}$ from $50-66,000 \mathrm{D}$ to $\sim 33-$ 35,000 (Fig. 5). Identical results were obtained with normal (Fig. 5, lanes 1 and 2) and JP PMN FPR (Fig. 5, lanes 3 and 4), 

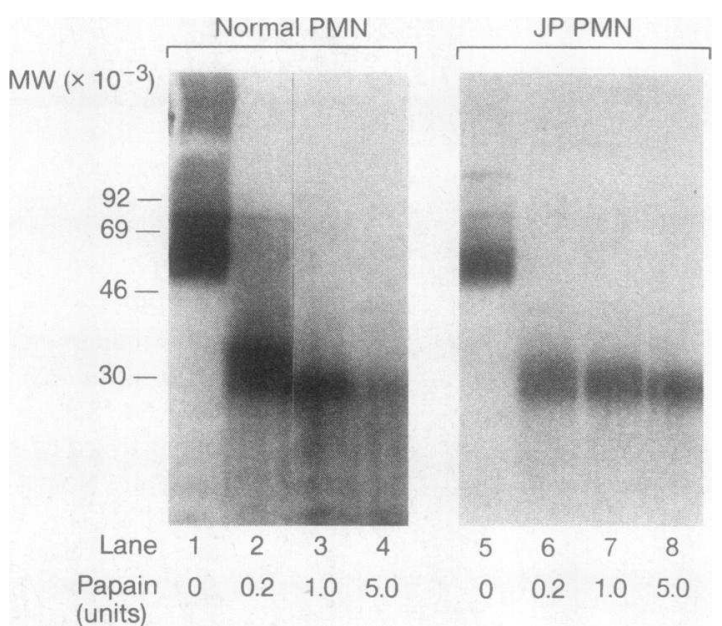

Figure 6. Autoradiograms demonstrating the ability of papain to digest normal and JP PMN ${ }^{125}$ I-FPR. Solubilized ${ }^{125}$ I-FPR preparations ( $40 \mu \mathrm{g}$ protein each) were incubated for $2 \mathrm{~min}$ at $37^{\circ} \mathrm{C}$ with increasing concentrations of papain. Reactions were terminated and material processed as described in Methods. (Lane 1) normal PMN ${ }^{125}$ I-FPR, no papain; (lane 2) normal PMN ${ }^{125} \mathrm{I}-\mathrm{FPR}$ plus 0.2 $\mathrm{U}$ of papain; (lane 3) normal PMN ${ }^{125} \mathrm{I}-\mathrm{FPR}$ plus $1.0 \mathrm{U}$ of papain; (lane 4) normal PMN ${ }^{125}$ I-FPR plus 5.0 U of papain; (lane 5) JP PMN ${ }^{125} \mathrm{I}-\mathrm{FPR}$, no papain; (lane 6) JP PMN ${ }^{125} \mathrm{I}-\mathrm{FPR}$ plus $0.2 \mathrm{U}$ of papain; (lane 7) JP PMN ${ }^{125}$ I-FPR plus $1.0 \mathrm{U}$ of papain; and (lane 8) JP PMN ${ }^{125} \mathrm{I}-\mathrm{FPR}$ plus $5.0 \mathrm{U}$ of papain.

indicating that JP PMN FPR exhibited a similar degree of glycosylation as normal PMN FPR.

Next, we performed experiments designed to examine the protein backbone of JP PMN FPR. Normal PMN ${ }^{125}$ I-FPR can be digested with papain (by limited digestion) to a species exhibiting a $M_{\mathrm{r}}$ of 32,000 (that retains the cross-linked labeled peptide) $(18,19)$. Detergent extracts of affinity-labeled normal and JP PMN FPR (containing identical amounts of protein) were incubated $\left(37^{\circ} \mathrm{C}, 2 \mathrm{~min}\right)$ with increasing concentrations of activated papain. Reactions were terminated by the addition of equal volumes of cold buffer containing $2.0 \mathrm{mM}$ cystatin. After digestion, samples were subjected to SDS-PAGE followed by autoradiography (Fig. 6). Incubation of affinity-labeled normal PMN FPR with $0.2 \mathrm{U}$ papain decreased its $M_{\mathrm{r}}$ from $55-68,000 \mathrm{D}$ (Fig. 6, lane 1 ) to a labeled species exhibiting a $M_{\mathrm{r}}$ of 28-35,000 (Fig. 6, lane 2). When $1.0 \mathrm{U}$ of papain was used, its $M_{\mathrm{r}}$ decreased further to $28-30,000 \mathrm{D}$ (Fig. 6, lane 3) and radioactivity was detected at the bottom of the gel (i.e., degraded ${ }^{125}$ I-FPR). Incubation with $5.0 \mathrm{U}$ of papain resulted in almost complete digestion of normal PMN FPR (Fig. 6, lane 4). Incubation of affinity-labeled JP PMN FPR with $0.2 \mathrm{U}$ of papain decreased its $M_{\mathrm{r}}$ to $28-32,000 \mathrm{D}$ (Fig. 6, lane 6). In contrast to normal PMN FPR, however, incubation of JP PMN FPR with either 1.0 or $5.0 \mathrm{U}$ of papain did not result in further digestion (Fig. 6, lanes 7 and 8 ). Complete digestion of JP PMN FPR could be achieved when $20 \mathrm{U}$ of papain was used (not shown). Thus, it appears that JP PMN FPR is more resistant to papain cleavage than normal PMN FPR. One possible explanation for these findings was that normal and JP PMN FPR may differ in their amino acid composition. If so, it is possible that the peptide(s) representing FPR's would exhibit different isoelectric points. To examine this possibility, detergent extracts obtained from affinity-labeled normal and JP PMN FPR (equal amounts of protein and radioactivity) were subjected to 2D-PAGE, followed by autoradiography (Fig. 7). The first dimension was run using a narrow $\mathrm{pH}$ gradient (3.76.2) to increase the chance of detecting small changes in pI. Coomassie-blue staining of 2D-PAGE performed using detergent extracts of affinity-labeled normal (Fig. $7 A$ ) and JP PMN (Fig. $7 \mathrm{~B}$ ) failed to detect differences between the two cell types. Autoradiogram of affinity-labeled normal PMN extract 2DPAGE revealed the presence of four labeled bands, all of which exhibited the same molecular weight $(55-65,000 \mathrm{D})$ (Fig. 7 C). These bands (referred to as 1 to 4 ) had pl's of $1=5.2,2=5.4,3$ $=5.85$, and $4=6.1$. Autoradiogram of affinity-labeled JP PMN extract 2D-PAGE revealed the presence of bands 1, 3, and 4 (Fig. 7 D). Band 2 was barely detectable. To analyze
A.

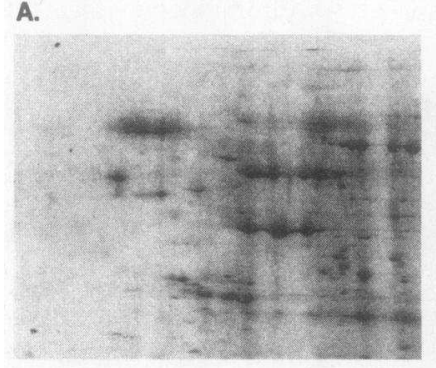

B.

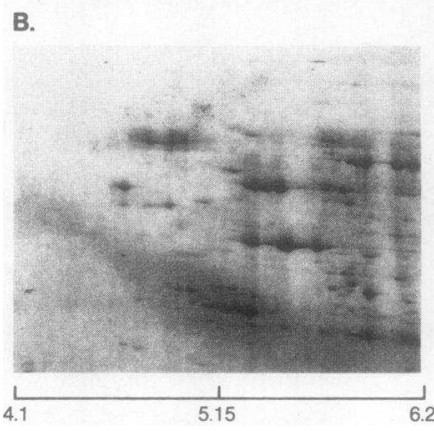

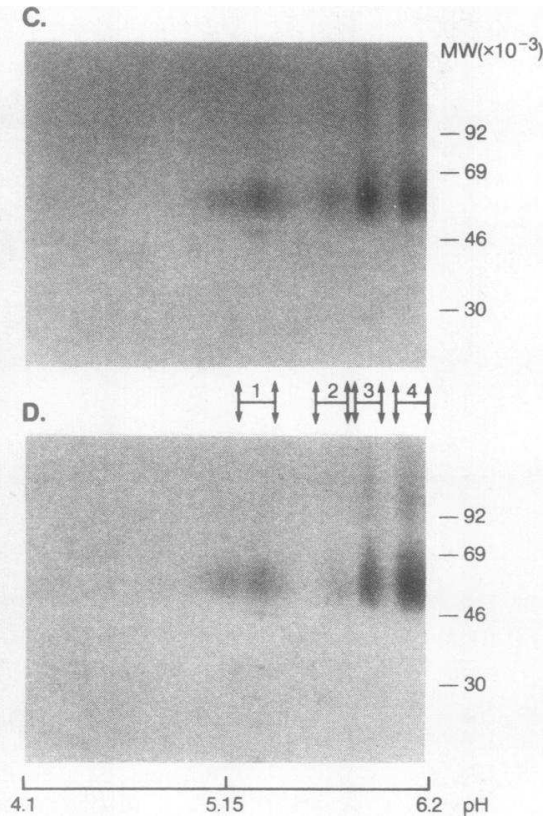

Figure 7. Coomassie-blue stain (2D-PAGE) of $(A)$ normal PMN ${ }^{125}$ I-FPR detergent extract; and $(B)$ JP PMN ${ }^{125} \mathrm{I}$ FPR detergent extract. Autoradiograms of $(C)$ normal PMN ${ }^{125}$ I-FPR gel shown in $A$; and $(D)$ JP PMN ${ }^{125}$ I-FPR gel shown in $B$. Each gel contained $200 \mu \mathrm{g}$ of total protein (applied in the first dimension). Total cpm applied: normal PMN ${ }^{125}$ I-FPR = 59,089; JP PMN ${ }^{125}$ I-FPR $=57,379$. Both preparations were run simultaneously in the first and second dimension. Second dimension was performed using $7-17 \%$ gradient slab gels. 
further this finding, autoradiograms obtained from normal and JP PMN were subjected to soft laser densitometry. Densitometric analysis of JP PMN autoradiogram revealed a $30 \%$ diminution in band 1 and $85 \%$ diminution in band 2, as compared with normal PMN. Bands 3 and 4 were identical for the two cell types.

\section{Discussion}

Initially, we determined the ability of normal and JP PMN to migrate chemotactically upon stimulation with two well-characterized, pure chemotactic factors. JP PMN failed to respond chemotactically to FMLP (Fig. $1 A$ ) but exhibited normal chemotactic responses when highly purified human C5a was used (Fig. $1 B$ ). These results indicated that JP PMN were capable of responding chemotactically, and suggested that their inability to migrate to FMLP was stimulus specific and secondary to a proximal (i.e., receptor) defect. Interestingly, the defect was limited to chemotactic responsiveness since JP PMN were able to degranulate (Fig. $2 \mathrm{~A}$ ) and generate superoxide anion radicals in a normal fashion upon challenge with FMLP. It is unclear if similar findings (i.e., impaired chemotaxis with normal degranulation and superoxide anion generation) are common in JP. Previous studies using populations of these patients $(5,6$, 20) did not evaluate PMN function in a systematic manner.

To determine if JP PMN had a defect at the level of their formyl peptide receptor, we performed binding studies (under equilibrium conditions) to determine receptor number and $K_{\mathrm{d}}$ of binding (Fig. 3, text). Scatchard analysis of binding revealed that JP PMN had a $42 \%$ diminution in the number of high affinity FPR, as compared with normal PMN. Their $K_{d}$ 's of binding, however, were similar. Number of low affinity FPR was similar for both cell types. Thus, JP PMN had a diminished number of high affinity FPR. Taken together, the above findings are consistent with our previous work suggesting that high affinity FPR mediates formyl peptide-induced PMN chemotaxis $(12,15)$. Van Dyke et al. (6) reported diminished binding of ${ }^{3} \mathrm{H}$-FMLP by JP PMN, associated with a reduction in their chemotactic responsiveness to the ligand. These authors, however, did not discriminate between high and low affinity FPR.

To examine further JP PMN FPR, normal and JP PMN FPR were affinity labeled using ${ }^{125}$ I-FP and EGS, and detergent extracts analyzed by SDS-PAGE and autoradiography. Affinity-labeled JP PMN FPR exhibited the same approximate $M_{\mathrm{r}}$ as normal PMN FPR (Fig. 4). Furthermore, $N$-glycanase digestion studies demonstrated that affinity-labeled normal and JP PMN FPR exhibited a similar degree of glycosylation (Fig. 5). JP PMN ${ }^{125}$ I-FPR, however, was more resistant to limited papain digestion than normal PMN ${ }^{125}$ I-FPR (Fig. 6). Resistance to papain cleavage could be due to either a defect in the protein backbone of JP PMN FPR or to some change in its spatial configuration that might make the cleavage site(s) for papain less accesible.

Attempts were made to determine if JP PMN FPR exhibited significant changes in their amino acid composition. If so, it would be possible that JP PMN FPR's would exhibit different pl's than normal PMN FPR. Thus, detergent extracts of normal and JP PMN ${ }^{125}$ I-FPR were subjected to 2D-PAGE and examined by autoradiography. Both preparations were run at equal protein concentrations and contained similar amounts of total radioactivity, to allow a valid comparison. Analysis of normal PMN ${ }^{125}$ I-FPR by 2D-PAGE and autoradiography demonstrated the presence of four isoforms (Fig. $7 \mathrm{C}$ ). These isoforms ranged in $\mathrm{pI}$ from 5.2 to 6.1. Previous determinations of ${ }^{125} \mathrm{I}$-FPR $\mathrm{pI}$ were performed using a pH gradient from 3 to 10 (18). Under these conditions two broad bands were detected by autoradiography, exhibiting pl's of 5.8 and 6.2 (18). Under the conditions used here a narrower $\mathrm{pH}$ gradient was generated in the first dimension, allowing a finer discrimination of pl's. We detected a 5.85 and a 6.1 isoform but previously unreported isoforms (pl's 5.2 and 5.4) were also present (Fig. $7 \mathrm{C}$ ). All these isoforms were specifically labeled, since they were not detected when crosslinking of FPR was performed in the presence of 100 -fold excess unlabeled peptide. It should be noted that for these studies, PMN from single donors (i.e., not pooled) were used. These four FPR isoforms appear to be constant when different preparations $(n=24)$ of normal PMN ${ }^{125}$ I-FPR are used.

Interestingly, 2D-PAGE and autoradiography of JP PMN ${ }^{125}$ I-FPR revealed the presence of bands 1,3 , and 4 (i.e., pl's $5.2,5.85$, and 6.1, respectively). Band 2 was almost absent. Furthermore, densitometric analysis of autoradiograms demonstrated that JP PMN had a 30\% diminution in band 1 and an $85 \%$ diminution in band 2 . Since both preparations were obtained from an equal number of PMN, and run under equal protein concentrations (and total radioactivity), we believe that these findings actually represent changes in amounts of JP PMN ${ }^{125}$ I-FPR. The fact that bands 1,3 , and 4 migrated with almost identical pI's as normal PMN ${ }^{125}$ I-FPR does not mean that their composition is identical since small changes in either glycosylation or a point mutation (and substitution with a similarly charged amino acid) would not be detected. If their amino acid composition is similar to normal PMN FPR, then their resistance to papain cleavage could be explained either by changes in spatial configuration or by the way the JP PMN FPR is inserted in the plasma membrane. The former possibility may be relevant if JP PMN FPR differs from normal PMN FPR in their oligosaccharide chain. Thus, although JP PMN FPR exhibited a diminution in bands 1 and 2, current data does not allow discrimination of the putative defect.

In summary, these studies represent the first reported characterization of JP PMN FPR and results suggest that the abnormal chemotactic response to formyl peptides exhibited by these cells is due, at least in part, to an abnormal population of FPR's. Current efforts are directed at cloning the normal PMN FPR's. If successful, we should be able to characterize the molecular defect exhibited by JP PMN FPR's.

\section{Acknowledgments}

This work was supported by grants from the National Institutes of Health (AM-28566, DE-08138, AI-28290, and DE-07245).

\section{References}

1. Showell, H. J., R. J. Freer, S. H. Zigmond, E. Schiffman, S. Aswanikumar, B. Corcoran, and E. L. Becker. 1976. The structure-activity relations of synthetic peptides as chemotactic factors and inducers of lysosomal enzyme secretion for neutrophils. J. Exp. Med. 143:1154-1169.

2. Simchowitz, L., and I. Spilberg. 1979. Generation of superoxide radicals by human peripheral neutrophils activated by chemotactic factor. Evidence for the role of calcium. J. Lab. Clin. Med. 93:583-593.

3. Williams, L. T., R. Snyderman, M. C. Pike, and R. J. Lefkowitz. 1977. Specific receptor sites for chemotactic peptides on human polymorphonuclear leukocytes. Proc. Natl. Acad. Sci. USA. 74:1204-1208. 
4. Page, R. C., and H. E. Schroeder. 1976. Pathogenesis of inflammatory periodontal disease. A summary of current work. Lab. Invest. 34:235-242.

5. Van Dyke, T. E., H. U. Horoszewicz, L. J. Cianciola, and R. J. Genco. 1980. Neutrophil chemotaxis dysfunction in human periodontitis. Infect. Immun. 27:124-132.

6. Van Dyke, T. E., M. J. Levine, L. A. Tabak, and R. J. Genco. 1981. Reduced chemotactic peptide binding in juvenile periodontitis: A model for neutrophil function. Biochem. Biophys. Res. Commun. 100:1278-1284.

7. Haffajee, A. D., S. S. Socransky, J. L. Ebersol, and D. J. Smith. 1984 Clinical, microbiological and immunological features associated with the treatment of active periodontosis lesions. J. Clin. Periodontol. 11:600-615.

8. Perez, H. D., F. Elfman, D. Chenoweth, and C. Hooper. 1986. Preparation and characterization of a derivative of wheat germ agglutinin that specifically inhibits polymorphonuclear leukocyte chemotaxis to the synthetic chemotactic peptide $N$-formyl-methionyl-leucyl-phenylalanine. J. Immunol. 136:1813-1819.

9. Perez, H. D., M. Lipton, and I. M. Goldstein. 1978. A specific inhibitor of complement (C5)-derived chemotactic activity in serum from patients with systemic lupus erythematosus. J. Clin. Invest. 62:29-38.

10. Zigmond, S. H., and J. G. Hirsch. 1973. Leukocyte locomotion and chemotaxis: new methods for evaluation and demonstration of a cell-derived chemotactic factor. J. Exp. Med. 137:387-410.

11. Niedel, J. S., S. Wilkinson, and P. Cuatrecasas. 1979. Receptor-mediated uptake and degradation of ${ }^{125}$ I-chemotactic peptide by human neutrophils. $J$ Biol. Chem. 254:10700-10706.

12. Perez, H. D., F. Elfman, and E. Lobo. 1987. Removal of human polymor- phonuclear leukocyte surface sialic acid inhibits reexpression (or recycling) of formyl peptide receptors. J. Immunol. 139:1978-1984.

13. O'Farrell, P. A. 1975. High resolution two-dimensional electrophoresis of proteins. J. Biol. Chem. 250:4007-4021.

14. Perez, H. D., E. Kelly, D. Chenoweth, and F. Elfman. 1988. Identification of the C5a des Arg cochemotaxin. Homology with vitamin D-binding protein (group-specific component globulin). J. Clin. Invest. 82:360-363.

15. Perez, H. D., F. Elfman, S. Marder, E. Lobo, and H. E. Ives. 1989. Formyl peptide-induced chemotaxis of human polymorphonuclear leukocytes does not require either marked changes in cytosolic calcium or specific granule discharge. Role of formyl peptide receptor reexpression (or recycling). J. Clin. Invest. 83:1963-1970.

16. Zigmond, S. H. 1981. Consequences of chemotactic peptide receptor modulation for leukocyte orientation. J. Cell Biol. 88:644-647.

17. Koo, C., R. J. Lefkowitz, and R. Snyderman. 1982. The oligopeptide chemotactic factor receptor on human polymorphonuclear leukocyte membranes exists in two affinity states. Biochem. Biophys. Res. Commun. 106:442449.

18. Malech, H. L., J. P. Gardner, D. F. Heiman, and S. Rosenzweig. 1985. Asparagine-linked oligosaccharides on formyl peptide chemotactic receptors of human phagocytic cells. J. Biol. Chem. 260:2509-2514.

19. Dolmatch, B., and J. Niedel. 1983. Formyl peptide chemotactic receptor: evidence for an active proteolytic fragment. J. Biol. Chem. 258:7570-7577.

20. Clark, R. A., R. C. Page, and G. Wilde. 1977. Defective neutrophil chemotaxis in juvenile periodontitis. Infect. Immun. 18:694-700. 